\title{
Multimodal in vivo imaging reveals limited allograft survival, intrapulmonary cell trapping and minimal evidence for ischemia-directed BMSC homing
}

Bert R Everaert ${ }^{1,2,5^{*}}$, Irene Bergwerf ${ }^{3}$, Nathalie De Vocht ${ }^{3,4}$, Peter Ponsaerts ${ }^{3}$, Annemie Van Der Linden ${ }^{4}$, Jean-Pierre Timmermans ${ }^{1}$ and Christiaan J Vrints ${ }^{2}$

\begin{abstract}
Background: Despite positive reports on the efficacy of stem cell therapy for the treatment of cardiovascular disease, the nature of stem cell homing to ischemic tissues remains elusive.

Results: We used a mouse model of peripheral tissue ischemia to study the survival and homing capacity of dual reporter gene (eGFP/Luciferase) expressing bone marrow-derived stromal cells (BMSC). Cell homing and survival were studied in the presence and absence of ciclosporin A (CSA) immunosuppression using bioluminescence imaging (BLI) together with confocal endomicroscopy. Different injection strategies were applied: central venous $(\mathrm{CV})$, intra-arterial (IA) and intramuscular (IM). BLI and confocal endomicroscopy evidenced complete rejection of the IM injected allogeneic BMSC transplant within 5 to 10 days. Immunosuppression with CsA could only marginally prolong graft survival. IM injected BMSC did not migrate to the site of the arterial ligation. CV injection of BMSC resulted in massive pulmonary infarction, leading to respiratory failure and death. Intrapulmonary cell trapping was evidenced by confocal endomicroscopy, BLI and fluorescence microscopy. IA injection of BMSC proved to be a feasible and safe strategy to bypass the lung circulation. During the follow-up period, neither BLI nor confocal endomicroscopy revealed any convincing ischemia-directed homing of BMSC.
\end{abstract}

Conclusions: BLI and confocal endomicroscopy are complementary imaging techniques for studying the in vivo biology of dual reporter gene-expressing BMSC. Allogeneic BMSC survival is limited in an immunocompetent host and cannot be preserved by CsA immunosuppression alone. We did not find substantial evidence for ischemia-directed BMSC homing and caution against CV injection of BMSC, which can lead to massive pulmonary infarction.

Keywords: Stem cell, BMSC, Homing, Bioluminescence, Confocal endomicroscopy

\section{Background}

Mesenchymal stem cell (MSC) therapy is currently being explored in almost any clinical field involving tissue restoration or modulation of immune responses [1]. This widely applicable, simple and straightforward therapy would represent a milestone in modern medicine. The

\footnotetext{
*Correspondence: berteveraert@me.com

'Laboratory of Cell Biology and Histology, University of Antwerp, Antwerp, Belgium

'Laboratory of Cellular and Molecular Cardiology, Antwerp University

Hospital, Antwerp, Belgium

Full list of author information is available at the end of the article
}

initial enthusiasm about the promising results of MSC therapy in preclinical animal studies, and later on also in phase 1 and phase 2 clinical trials was subsequently tempered by the outcome of larger randomized, placebo-controlled trials. The reasons herefore are not completely understood and probably relate to our lack of knowledge and understanding of the complex nature of MSC biology.

MSC were first described as clonogenic fibroblast precursor cells, residing in mouse bone marrow, thymus and spleen [2]. After 30 years of MSC research, the International

\section{Biomed Central}


Society for Cellular Therapy, in an effort to facilitate data exchange between investigators, came up with a set of minimal criteria to define human MSC. These cells should be plastic-adherent when maintained in standard culture conditions, have multilineage differentiation potential into osteoblasts, adipocytes and chondroblasts in vitro and express a specific set of cellular markers.

Despite the consensus on in vitro MSC characteristics, many unresolved questions remain about MSC biology in vivo, precluding its potential clinical applicability on a large scale [1,3]. Key questions on whether MSC truly undergo lineage differentiation and on the mechanisms through which these cells act on target tissues, i.e., their anti-apoptotic, pro-angiogenic or immune modulatory properties, are still matter of intense debate. Although many animal studies reported the positive effects of MSC therapy, usually very little information is provided about the long-term survival and engraftment of the administered MSC. A plausible explanation for this lack of information might be the absence of an easily applicable "gold standard" for studying cell survival and homing in vivo. Indeed, whole-body imaging techniques using radioactive or magnetic labeling dyes, fluorescent markers or reporter genes, all have disadvantages and probably a combination of different imaging modalities is needed to provide a reliable picture.

In addition, uncertainty remains about the optimal transplantation route, time frame and cell dose, and whether homing after systemic infusion is an active rather than passive "cell entrapment" phenomenon $[4,5]$. Finally, the safety of injecting vast amounts of foreign cells is a matter of concern, and although a relatively large amount of safety data has been gathered from phase 1 and 2 clinical trials, the observation that many systemically infused MSC become entrapped in the lungs warrants further investigation [6].

In the present study we combined two complementary in vivo imaging techniques, i.e., bioluminescence imaging
(BLI) and confocal endomicroscopy, to study dual reporter gene-expressing stem cell suvival and migration towards an ischemic stimulus in vivo.

\section{Results}

\section{Survival and migration characteristics of locally injected} BMSC

To study the in vivo survival and migration characteristics of IM injected BMSC, cells were transplanted into the calf muscles $24 \mathrm{~h}$ after induction of hindlimb ischemia. Prior to injection, eGFP fluorescence intensity and Luciferase activity were checked by confocal endomicroscopy and bioluminescence imaging methods, respectively (Figure 1$)$. Mean $( \pm$ SD) cell diameter was $13.4 \mu \mathrm{m}$ $( \pm 3.5)$. The biology of the cell transplant was investigated during a 3-week follow-up period using both in vivo imaging techniques. The high optical resolution (micrometer scale) of confocal endomicroscopy enabled us to perform a detailed study of the survival and differentiation characteristics of single cells in the BMSC transplant and to monitor BMSC homing towards the ischemic site. BMSC could be seen as a sharp delineated tissue infiltrate at baseline. The transplanted cells developed a spindle-shaped pattern with cell branches within a few days. The transplant appeared to be stable throughout the first week but then displayed a rapid and steep drop in BMSC number and density during the second week. As a result of the rejection of the BMSC transplant, cellular morphology became more heterogeneous in both size and appearance, less densely organized and more fragmented. BLI signals were in accordance with endomicroscopic findings (Figure 2). Signal intensity and position of the cell transplant remained fairly stable in the first week after transplantation. Hereafter, BLI signals rapidly faded to background levels in all animals during the second week after transplantation. Using BLI to discern active homing of the

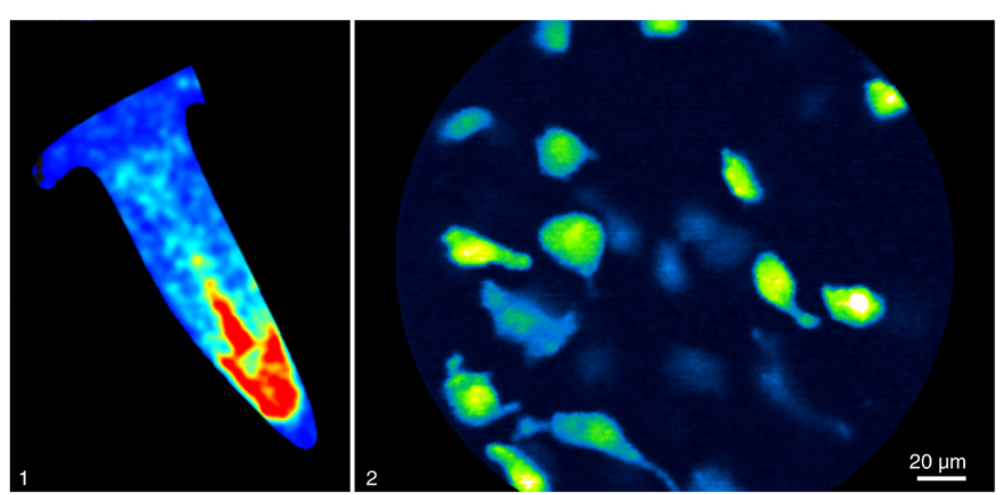

Figure 1 Baseline expression of reporter genes before BMSC cell injection. 1: Luciferase expression of BMSC measured with bioluminescence imaging. 2: In vivo endomicroscopy images of eGFP expressing BMSC before injection. 


Figure 2 Survival of IM injected BMSC visualized with bioluminescence (upper row) versus confocal endomicroscopy (lower row).
Immediately after IM injection a cell infiltrate was seen at the injection site. In the absence of immunosuppression, in most animals no residual
cells were detectable beyond 8 days after cell transplantation. BMSC survival in mice treated with CsA was only temporarily prolonged. At day 8
post-transplantation some residual, viable cells were still observed, but the signal weakened rapidly during the second week after transplantation.
No cell infiltrate was detected beyond 10 days after transplantation.

cell transplant towards the ischemic site (which is somewhat difficult because of the low optical resolution $( \pm 1 \mathrm{~mm})$ of the BLI technique), we did not observe much change in the position of the cell transplant over time, nor was there evidence for active homing towards the ischemic site. To study the homing capacity of individual BMSC, we applied confocal endomicroscopy to the site of tissue ischemia. Here, the day after transplantation, only one eGFP positive cell could be found in the ischemic muscular region in only one transplanted animal. However, at all later time points, confocal endomicroscopy did not demonstrate homing of eGFP ${ }^{+}$BMSC towards the area surrounding the ligation site Table 1.

\section{Ciclosporin immune suppression cannot sustain prolonged BMSC survival}

To study whether BMSC rejection could be delayed, we studied the rejection process of IM injected BMSC in animals treated with or without daily IP injections of CsA ( $n=8$ for both groups). Using BLI and confocal endomicroscopy, we were able to show that this immunosuppressive regimen only marginally prolonged BMSC transplant

Table 1 Comparison of detection techniques

\begin{tabular}{lll}
\hline & Bioluminescence imaging & Confocal endomicroscopy \\
\hline Strength & Non-invasive & High spatial resolution \\
& Low background signals & Single cell imaging \\
& Highly specific for & Cell morphology studies \\
& Luciferase activity & \\
\hline Weakness & Low spatial resolution & Invasive procedure \\
& Low cell numbers & Requires direct \\
& not detectable & probe contact \\
& Limited tissue & Autofluorescence \\
& penetration & reduces specificity \\
& Superior specificity & Superior spatial resolution \\
\hline
\end{tabular}

survival, a difference that was not statistically significant $(p=0.14)$ (Figure 3). To exclude the possibility of underimmunosuppression, a higher dose of CsA $(30 \mathrm{mg} / \mathrm{kg} / \mathrm{d}$ instead of $10 \mathrm{mg} / \mathrm{kg} / \mathrm{d}$ ) was used in about half of the animals. No apparent difference in BMSC transplant survival was observed between mice treated with either high- or lowdose immunosuppression.

\section{Intrapulmonary cell trapping of BMSC}

$\mathrm{CV}$ injection of BMSC by right heart cannulation resulted in respiratory distress and led to respiratory failure and death within minutes (5/5). Confocal endomicroscopy on resection specimens revealed trapped $\mathrm{eGFP}^{+} \mathrm{BMSC}$ in both lungs, the right ventricle and the right atrium. Intrapulmonary cell trapping was confirmed by BLI imaging 30 min after injection of D-luciferin pre-incubated BMSC. Bioluminescence signals were clearly visible in both lungs and were restricted to the right side of the heart. No BLI signal could be detected in the left atrium or the left ventricle, nor in tissue specimens of liver, kidney, spleen or

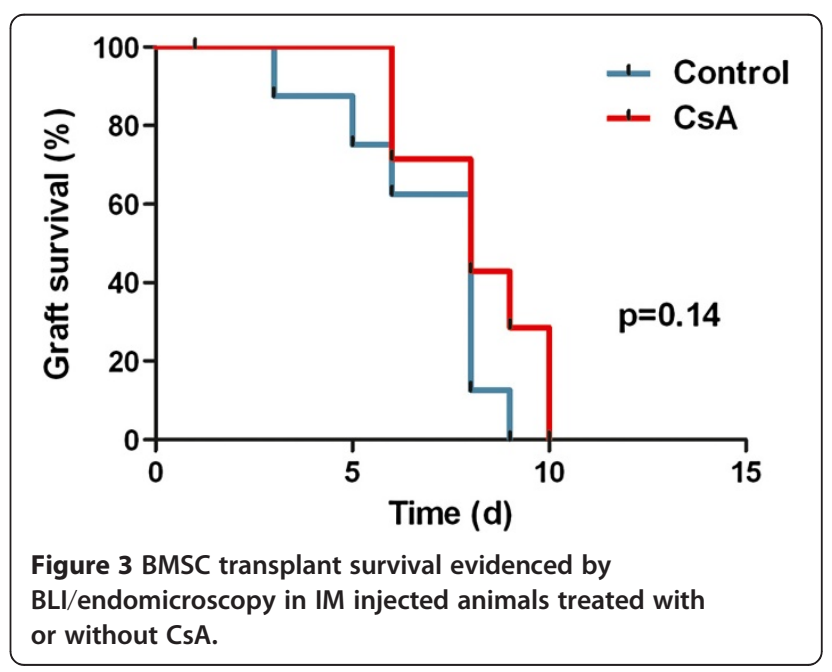


blood (Figure 4). Intrapulmonary cell trapping after CV BMSC injection was further evident on fluorescence microscopic images of formalin-fixed, paraffin-embedded lung slices. $\mathrm{eGFP}^{+}$cells were seen in small vessels or capillaries throughout the entire lung (Figure 5). Some cells appeared as round cells, while others displayed cell processes and seemed to evade the pulmonary circulation and enter into the lung parenchyma.

\section{Minimal evidence for BMSC homing after systemic transplantation}

To study the process of ischemia-directed cell homing, BMSC were injected directly into the arterial circulation $(n=5)$ by direct injection into the left heart ventricle, as such bypassing the pulmonary circulation. This procedure proved to be feasible and safe. During a two-week followup period, neither BLI nor confocal endomicroscopy demonstrated any convincing sign of active migration of BMSC towards the ischemic site in an immunocompetent host $(n=2)$. However, in one out of three animals treated with CsA $(10 \mathrm{mg} / \mathrm{kg} / \mathrm{d})$, a cluster of three BMSC was detected by confocal endomicroscopy in the same microscopic plain of the ischemic hindlimb (Figure 6, video sequence provided as Additional file 1). Taking into account their typical morphology, size and fluorescence intensity, we feel confident that these cells are indeed living BMSC. Whether these cells actively migrated to the ischemic site or whether their presence was merely a chance phenomenon due to passive cell entrapment, is impossible to deduce from this limited amount of study data. This small number of detected cells, together with the scarce evidence for BMSC homing in IM transplantation experiments, led us to conclude that active BMSC homing is a rare event.

\section{Discussion and conclusions}

In this study we investigated BMSC survival and ischemia-directed homing using two in vivo reporter gene expression imaging techniques, BLI and confocal endomicroscopy. Endomicroscopy has superior sensitivity in visualizing transplants at low cellular concentrations or with fading tracer signals. Its high optical resolution enables the study of cellular morphology, delineation of the cell infiltrate as well as homing capacity of individual cells. Drawbacks of the technique are its invasive nature requiring direct tissue contact with limited deep tissue penetration. Autofluorescence did occur at times but the chance of false positive findings in our study was rather

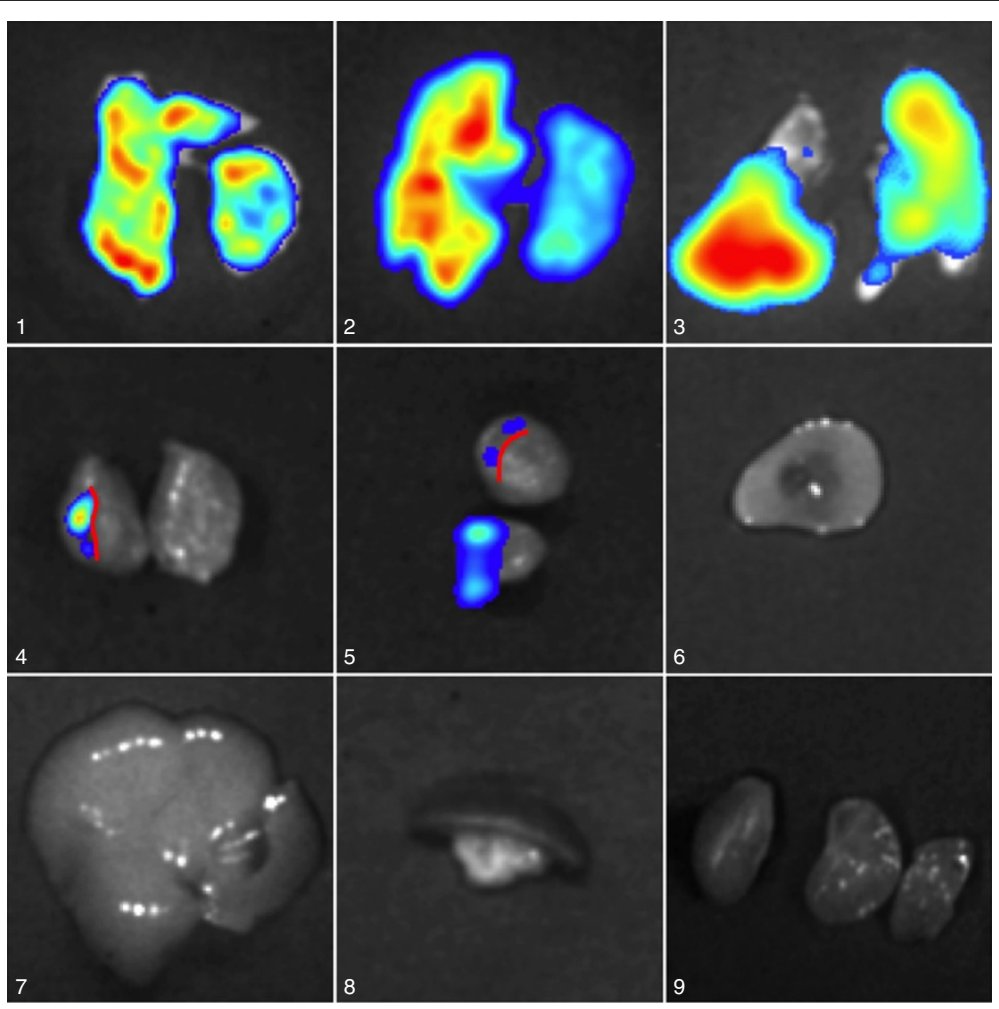

Figure 4 BLI imaging of dissection specimens 30 min after CV injection of BMSC. 1-5: Bioluminescent BMSC are present throughout the entire lung (3 independent experiments) and in the right heart ventricle (longitudinal (4) and cross-section (5), red bar depicts the interventricular septum). Note that the left ventricle is devoid of luminescent cells. 6-9: No signals are observed in the blood (6), or in other organs (liver (7); spleen (8); kidneys (9)). 

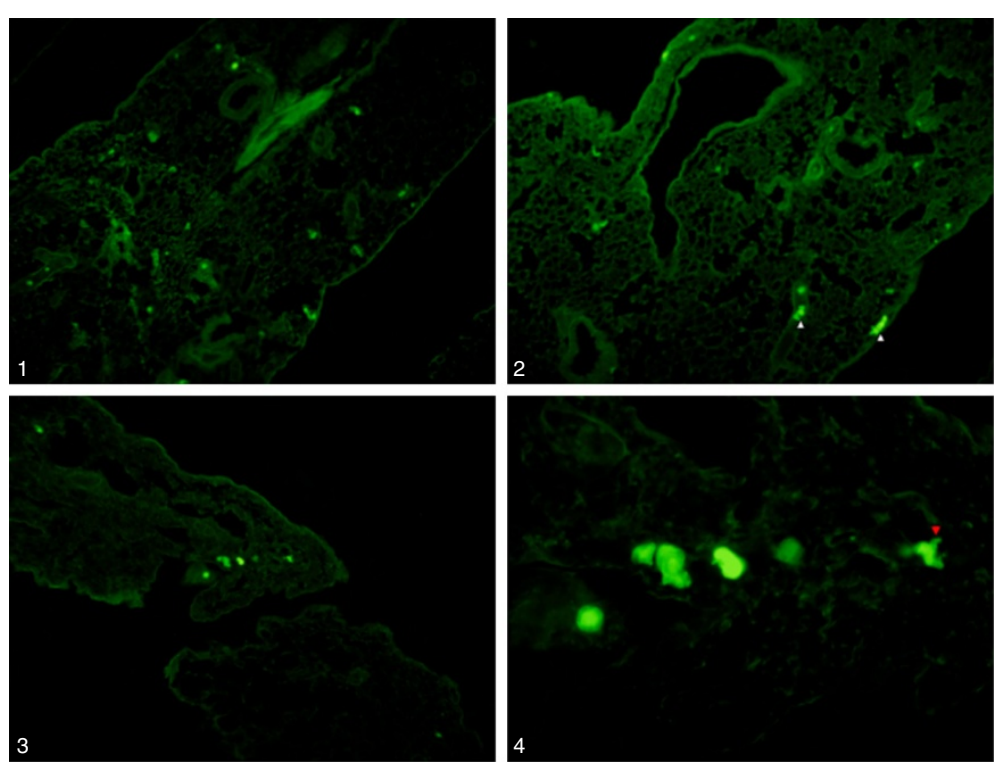

Figure 5 Lung entrapment of BMSC. 1-3: Fluorescence microscopic images of lung slices show eGFP ${ }^{+} \mathrm{BMSC}$ as microemboli throughout the entire lung. Cells and cell aggregates can be seen in vascular structures (10x). 4: Higher magnification of image 3. Intravascular cell entrapment and diapedesis into the lung parenchyma (red arrowhead) of CV injected BMSC (40X).

limited because of the typical cell size and morphology of BMSC. BLI, on the other hand, is a non-invasive and hence more practical technique to use in follow-up studies of transplant survival, but it lacks the high optical resolution of endomicroscopy. It also has superior specificity for detecting Luc ${ }^{+}$cells. However, BLI is unable to detect single cells or transplants with low cell concentration or limited cell numbers. Nevertheless, a combination of the high specificity of BLI joined with the high optical resolution of confocal endomicroscopy, overcomes several constraints of either technique alone and enables to study the biology of BMSC survival and homing with more precision and accuracy.

Studying cell homing using reporter genes has several advantages over conventional cell tracking agents, such as cell labeling dyes, microparticles, radioisotopes or magnetic resonance imaging (MRI) contrast agents. An important drawback of these conventional methods is that the agent used can become decoupled from the original cell transplant and/or ingested by other cell types, such as macrophages. Macrophage ingestion is a major confounder in transplantation studies because many

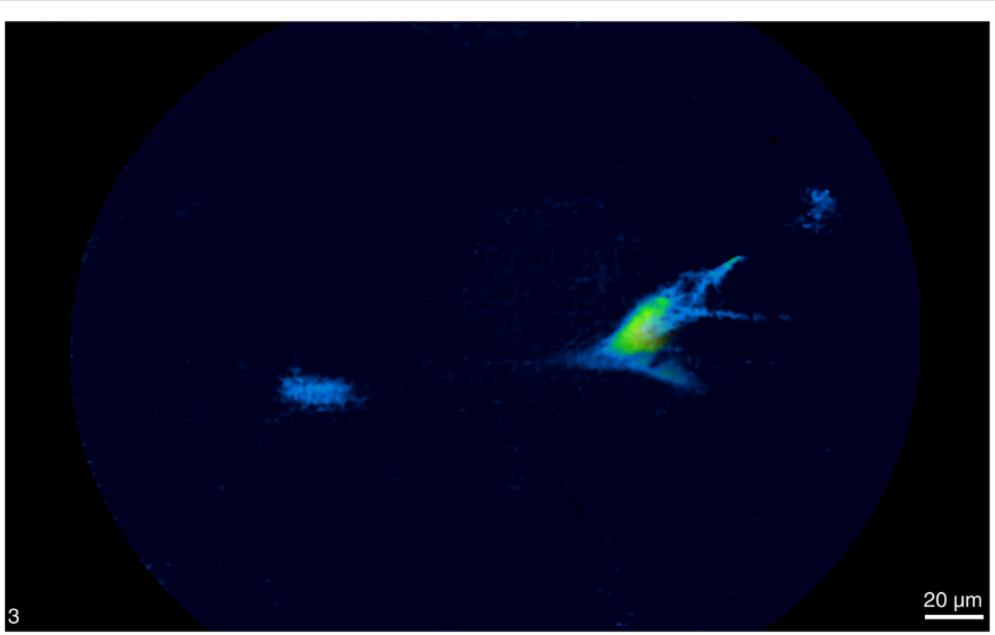

Figure 6 Ischemia-directed homing of BMSC after intra-arterial injection. Confocal endomicroscopic image of three BMSC at the ischemic site. Of note are the typical BMSC cell size, cellular morphology and $\mathrm{EFP}^{+}$fluorescence indicating living cells. 
cells of donor origin die shortly after cell transplantation, triggering a local immune reaction with macrophage infiltration [7].

Because reporter genes are integrated into the cell genome, cell tracking studies using reporter gene expression is much less likely to yield false positive results: the expression of the reporter gene is limited to viable cells of the original cell transplant and is preserved in successive cell divisions. When cells do not survive or are ingested by other cells, the signal diminishs over time. However, since the reporter gene is integrated in the genomic DNA, cell proliferation will increase rather than decrease signal intensity [4], as such preventing dilution of the original labeling signal.

Despite these benefits, the use of reporter genes also entails a number of caveats. A first problem could arise from the induction of immune responses directed against non-host alloantigens expressed on the cell surface of the transfected cells that express non-self proteins, such as Luc or eGFP. However, Bergwerf et al. [8] demonstrated that the immune reactive cytotoxic T-cell response following BMSC transplantation was not specific for the non-self eGFP or Luc genes. These authors suggested that the observed T-cell mediated transplant rejection could be due to the presence of xenogeneic constituents of the growth medium, such as fetal calf and horse serum, which render the cultured cells more immunogeneic. Another concern is gene silencing, which is a mechanistically very complex phenomenon that can vary with specific experimental conditions due to a site-dependent adaptation of the expression of the reporter gene in vivo [9].

We demonstrated in our hindlimb ischemia mouse model that cell transplant survival after IM injection was limited in time and that CsA immunosuppression alone was not sufficient to maintain long-term survival of the allogenic BMSC graft. Our data are in line with the BLI results of Swijnenburg et al [10]. In this study, immunosuppression with mycophenolate mofetil, sirolimus or tacrolimus, both in monotherapy or in combination, failed to induce long-term survival of embryonic stem cell xenografts. On the other hand, Swanger et al. [11] showed that, at least in the central nervous system, high dose CsA significantly increased allogeneic BMSC graft survival compared to standard dose CsA, indicating that higher doses of CsA could be sufficient to maintain long-term survival of allogeneic BMSC at immunologically privileged sites. Furthermore, evidence from BLI experiments from Zangi et al. [12] about survival and rejection of IV or intraperitoneally injected allogeneic MSC transplants, indeed showed that, at least in mice, survival of a systemically injected MSC transplant is limited in time. Moreover, it was demonstrated that even IM injection of BMSC in immune competent syngeneic mice did not result in permanent graft survival [8].
We used a hindlimb ischemia model for the study of ischemia-directed homing firstly for reasons of good accessibility of the tissues of interest for the optical probe of the confocal endomicroscope. Therefore, in vivo endomicroscopy would be less suitable to study homing towards deeper anatomical structures or to moving organs, such as the heart. Secondly, hindlimb ischemia mouse models have already been validated extensively as experimental set-up for the study of ischemia-directed MSC homing. A better understanding of survival and homing characteristics of MSC in this experimental model is of particular relevance for the early-phase clinical trials on the use of human MSC for the treatment of critical limb ischemia that are currently ongoing [13].

We caution against $\mathrm{CV}$ injection of large amounts of BMSC because this leads to massive pulmonary infarction. Gao and co-workers already reported that systemic injection of MSC initially results in retention in the lungs, after which a gradual redistribution could be seen to the liver, bone marrow and other organs. These authors showed that the pulmonary retention could be reduced using the vasodilator sodium nitroprusside [14]. This finding, i.e. initial intrapulmonary cell trapping with gradual tissue redistribution, was confirmed by DaldrupLink et al. [15], who used MRI imaging, and by in vivo BLI studies by Schrepfer et al. [16]. In the latter study, episodes of tachypnea, apnea and hemodynamic alterations, characteristic of pulmonary embolism, were reported when MSC were directly infused into the inferior vena cava. Pulmonary entrapment was also evident in a myocardial infarction study with BrdU-labeled MSC [6]. When the lung circulation was bypassed through direct intracavitary administration of MSC, significantly more cells were observed within the infarction zone within the first hours after transplantation. In a later report by the same authors, long-term engraftment of the infused MSC, as initially suggested on the MRI images, could not be verified with Y chromosome gene detection. Further analysis revealed that the positive MRI signals originated from cardiac resident macrophages that had ingested the MRI contrast particles. Intriguingly, although cells of donor origin were absent at 4 weeks after infusion, ventricular function was still improved in the MSC-treated animals [17]. The authors attributed this phenomenon to possible paracrine effects. Another tissue distribution study in the clinical setting of myocardial infarction, showed initial lung uptake of intravenous (IV) injected ${ }^{18} \mathrm{~F}$-FDG-labeled bone marrow-derived cells, followed by a gradual redistribution to the reticuloendothelial system (spleen, liver, bone marrow) [18]. Stem cell homing towards the infarction site, however, could not be demonstrated. Evidence for the pulmonary entrapment of stem cells was also reported after IV injection of neural stem cells [19], leading to massive 
pulmonary inflammation and apoptosis. Together, these studies indicate that the lung might act as a barrier for the passage of different stem cell subtypes [20], especially for those that are relatively large and express abundant adhesion molecules [21]. Our study adds further evidence to the finding of intrapulmonary cell trapping after $\mathrm{CV}$ injection of BMSC and questions the safety of this route for BMSC transplantation.

As indicated by our results, direct intracavitary injection into the left ventricle is a feasible approach to circumvent this pulmonary "first-pass" effect, but it is rather impractical to use in small animal experiments. However, direct IA administration of BMSC in the arterial circulation, e.g. by intracoronary injection after myocardial infarction, thereby avoiding the lung circulation, would in our opinion prove to be the method of choice for BMSC cell application in large animal and human clinical trials. Furthermore, considering that active ischemia-directed BMSC homing of unstimulated BMSC was a rare biological phenomenon in our studies, this also favours direct IA injection of BMSC into the vasculature of the tissue of interest. Alternatively, the homing process could potentially be improved with use of stimulated rather than naive BMSC. BMSCs can easily be ex vivo manipulated by pretreatment, preconditioning and genetic modification, which was reported to increase their therapeutic benefit [22]. For instance, hypoxic proconditioning has been shown to stimulate the induction of several chemokine receptors, such as CXCR4 and CXCR7. This led to improved migration and recruitment of BMSC into ischemic tissues and stimulated secretion of proangiogenic and mitogenic factors improving therapeutic potential of the transplanted BMSC [23].

As a limitation of our work, we are aware that we could have underestimated the BMSC homing process because of the specific limitations of our detection techniques and experimental set-up. However, by combining the advantages of BLI with endomicroscopy, we feel confident about our results. However, it is possible that we underestimated the homing potential of BMSC, because of the limited graft survival time or the use of naive rather than stimulated or manipulated BMSC. Also, the use of CsA could have interfered with BMSC kinetics or altered the BMSC microenvironment, leading to an impaired homing respons. Transgenic immunodeficiency models, rather than immunosuppression with pharmacological agents, would be an interesting alternative to use in future studies on BMSC survival and homing. Finally, increasing the number of observations in each group would further strengthen our statements.

In summary, in this study a combination of BLI and confocal endomicroscopy was applied for dual reporter gene-expression imaging of BMSC survival and homing in a clinically relevant mouse model of peripheral tissue ischemia. We report that allogeneic BMSC graft survival time was limited in the ischemic muscle and that CsA immunosuppression alone was not able to sustain longterm survival of the allograft. We did not find abundant evidence for ischemia-directed BMSC homing as a common biological process and caution against the direct $\mathrm{CV}$ injection of large amounts of BMSC.

\section{Methods}

\section{Animals}

Homozygous ROSA26-L-S-L-Luciferase transgenic mice (FVB background) were purchased from Jackson Laboratories (strain 005125) and further bred in the specific pathogen-free animal facility of the University of Antwerp. Male offspring was used for BMSC culture. Male C57Bl/6 mice (Charles River) $(\mathrm{n}=26)$ were used as acceptors. Animals were kept in a normal day-night cycle (12/12) with free access to food and water. National and European principles of laboratory animal care were followed. All animal experimental procedures were approved by the Animal Care and Use Committee of the University of Antwerp (Permit Number: 2008-03).

\section{Reporter gene-expressing bone marrow-derived stromal cells (BMSC)}

BMSC genetically engineered with the Luciferase (Luc) and enhanced Green Fluorescent Protein (eGFP) reporter proteins were cultured and phenotypically characterized as previously described by Bergwerf et al. [8]. $\mathrm{eGFP}^{+} / \mathrm{Luc}^{+}$ BMSC were cultured in "complete expansion medium" (CEM) consisting of Iscove's modified Dulbecco's medium (Cambrex) supplemented with $8 \%$ fetal bovine serum (HyClone Laboratories), 8\% horse serum (Invitrogen), $100 \mathrm{U} / \mathrm{mL}$ penicillin (Invitrogen), $100 \mathrm{mg} / \mathrm{mL}$ streptomycin (Invitrogen), $0.25 \mathrm{mg} / \mathrm{mL}$ amphotericin B (Invitrogen). CEM was supplemented with $1 \mu \mathrm{g} / \mathrm{mL}$ Puromycine (Invivogen) to induce eGFP expression (eGFP-IRES-Pac cassette). Cultures were harvested twice a week using trypsin-EDTA (Invitrogen) and passaged at a 1:3 ratio in $15 \mathrm{ml}$ CEM in T75 culture flasks. Cells of passage number 5 to 20 were used in the experiments. Before transplantation, $\mathrm{eGFP}^{+} / \mathrm{Luc}^{+} \mathrm{BMSC}$ were washed twice in sterile PBS solution and resuspended at a final concentration of $5.10^{6} \mathrm{BMSC} / \mathrm{ml} \mathrm{NaCl}$ 0,9\%. Cell preparations were kept on ice until cell injection.

Hindlimb ischemia mouse model and cell transplantation Hindlimb ischemia was induced by ligation of the left femoral artery (LFA). In brief, 3- to 5-month-old male C57BL $/ 6$ mice were anesthetized (demetomidine $0.4 \mathrm{ml} / \mathrm{kg}$ and ketamine $37.5 \mathrm{mg} / \mathrm{kg}$, intraperitoneally). The LFA was ligated as close as possible to the left inguinal ligament using 7-0 polypropylene sutures $\left(\right.$ Prolene $^{\circledR}$, Ethicon, Johnson \& Johnson). Subsequently, the operation wound 
was closed and animals were placed under a heat source until full recovery. After $24 \mathrm{~h}$, mice were re-anesthetized and transplanted with $500.10^{3} \mathrm{eGFP}^{+} / \mathrm{Luc}^{+}$BMSC dissolved in $0.1 \mathrm{ml} \mathrm{NaCl} 0.9 \%$. The transplant route was either central venously $(\mathrm{CV})$ by right heart cannulation $(\mathrm{n}=5)$, intra-arterial (IA) by left ventricular cannulation $(n=5)$ or intramuscular (IM) directly into the calf muscle $(\mathrm{n}=16)$. For right and left heart cannulation, animals were intubated (23G catheter) and mechanically ventilated $\left(10 \mu \mathrm{l} / \mathrm{g}, 180 / \mathrm{min}, 2 \mathrm{~cm} \mathrm{H}_{2} \mathrm{O}\right.$ positive end-expiratory pressure) and a small parasternal thoracotomy was performed. Systemic injection was performed slowly at a rate of $100 \mu \mathrm{l} / \mathrm{min}$. To study the effects of the presence or absence of immunosuppressive therapy on immune rejection of the cell transplants, animals were treated with $(n=13)$ or without $(n=13)$ daily intraperitoneal injection of CsA. Two dosage regimens were compared: $10 \mathrm{mg} / \mathrm{kg} / \mathrm{d}$ $(\mathrm{n}=6)$ versus $30 \mathrm{mg} / \mathrm{kg} / \mathrm{d}(\mathrm{n}=7)$.

\section{Confocal endomicroscopy}

Homing of $\mathrm{eGFP}^{+} / \mathrm{Luc}^{+} \mathrm{BMSC}$ was visualized in vivo with the Cellvizio ${ }^{\circledR}$ LAB system (Mauna Kea Technologies) equipped with a $488 \mathrm{~nm}$ excitation laser. Cellvizio ${ }^{\circledR}$ LAB is a high-speed fluorescence microscope that permits real-time endoscopic fluorescence imaging through high-resolution fiber bundle micro-optics (UltraMini0 microprobe: tip diameter $2.6 \mathrm{~mm}$, lateral resolution $1.4 \mu \mathrm{m}$, field of view $240 \mu \mathrm{m}$, working distance $60 \mu \mathrm{m}$ ). In each mouse, cell homing was visualized 3 times per week, for a time period of 3 weeks. In brief, mice were anesthetized (demetomidine $0.4 \mathrm{ml} / \mathrm{kg}$ and ketamine $37.5 \mathrm{mg} / \mathrm{kg}$, intraperitoneally). A small skin incision was made in the inguinal region, the microprobe was advanced towards the ligation site and the underlying muscular tissues were scanned for the presence of fluorescent cells. A second incision at the level of the calf muscles enabled us to study the morphology, survival and homing characteristics of the IM injected cell infiltrate distal to the ligation site. Tissues were imaged for at least $5 \mathrm{~min}$ with continuous video recording. The contralateral hindlimb served as a negative control to discriminate $\mathrm{eGFP}^{+}$cell homing from autofluorescence background signals. After the procedure, the operation wounds were closed using 7-0 polypropylene sutures (Prolene ${ }^{\circledR}$, Ethicon, Johnson \& Johnson).

\section{In vivo bioluminescence imaging (BLI)}

In all transplanted mice, homing of viable $\mathrm{eGFP}^{+} / \mathrm{Luc}^{+}$ BMSC was analyzed with a non-invasive in vivo photonimager system (Biospace), three times weekly for 3 weeks (or less in case BLI signals consistently disappeared earlier). Mice were anesthetized (demetomidine $0.4 \mathrm{ml} / \mathrm{kg}$ and ketamine $37.5 \mathrm{mg} / \mathrm{kg}$, intraperitoneally) and intraperitoneally injected with $100 \mu \mathrm{l}$ D-luciferin
(30 mg $/ \mathrm{ml}$, dissolved in PBS) with a $29 \mathrm{G}$ needle. After $10 \mathrm{~min}$, bioluminescence signals were recorded for at least $10 \mathrm{~min}$. Bioluminescence recordings were layered onto a visual photographic image to create composite bioluminescence - photographic images. The data were analysed with Photovision software. To study the phenomenon of intrapulmonary cell trapping, CV injected BMSC were pre-incubated with D-Luciferin. Bioluminescence images of the dissected lungs, heart, spleen, kidneys, liver and blood were obtained postmortem.

\section{Histology - fluorescence microscopy}

Tissue specimens were fixed in $4 \%$ paraformaldehyde for $2 \mathrm{~h}$ at room temperature, washed 3 times with PBS and stored overnight in $70 \%$ ethanol. Tissues were paraffinembedded, sliced into $5 \mu \mathrm{m}$ thick tissue sections and analyzed for $\mathrm{eGFP}^{+}$cells using a Zeiss Axiophot fluorescence microscope equipped with an DP70 digital camera system (Olympus). Images were analyzed in $\mathrm{Cell}^{\mathrm{P}} \mathrm{Im}$ aging Software version 3.4 (Olympus).

\section{Statistical analysis}

Logrank test was used for curve comparison of survival data. A p-value of $<0.05$ was considered to be statistically significant.

\section{Additional file}

\section{Additional file 1: BMSC cluster.}

\section{Abbreviations}

BLI: Bioluminescence imaging; BMSC: Bone marrow stromal cell; CEM: Complete expansion medium; CsA: Ciclosporin A; CV: Central venous; eGFP: Enhanced green fluorescent protein; IA: Intra-arterial; IM: Intramuscular; IV: Intravenous; LFA: Left femoral artery; MRI: Magnetic resonance imaging; MSC: Mesenchymal stem cell; SD: Standard deviation.

\section{Competing interests}

The authors declare that they have no competing interests.

\section{Authors' contributions}

BRE carried out the animal studies, bioluminenscence, confocal endomicroscopy, histology, study design and drafted the manuscript. IB, NVD and PP provided BMSC, participated in study design and drafted the manuscript. AVDL provided BLI and confocal endomicroscopy and helped to draft the manuscript. JPT and CJV conceived of the study, participated in its design and coordination and helped to draft the manuscript. All authors read and approved the final manuscript.

\section{Acknowledgements}

The authors would like to acknowledge the efforts of D. De Rijck and D. Vindevogel in preparing this text. This work was supported by the Research Foundation - Flanders, grant No. G014906. B. Everaert and N. De Vocht are research assistants and $P$. Ponsaerts a post-doctoral fellow of the

Reseach Foundation - Flanders (FWO Flanders). The Cellvizio system was kindly provided by Mauna Kea Technologies (EC-FP6-NoE EMIL, LSHC-CT-2004-503569).

\section{Author details}

${ }^{1}$ Laboratory of Cell Biology and Histology, University of Antwerp, Antwerp, Belgium. ${ }^{2}$ Laboratory of Cellular and Molecular Cardiology, Antwerp 
University Hospital, Antwerp, Belgium. ${ }^{3}$ Laboratory of Experimental Hematology, Vaccine and Infectious Disease Institute (Vaxinfectio), University of Antwerp, Antwerp, Belgium. ${ }^{4}$ Biolmaging Laboratory, University of Antwerp, Antwerp, Belgium. ${ }^{5}$ Laboratory of Cell Biology and Histology, Groenenborgerlaan 171, Antwerp 2020, Belgium.

Received: 25 June 2012 Accepted: 11 November 2012

Published: 3 December 2012

\section{References}

1. Ankrum J, Karp JM: Mesenchymal stem cell therapy: Two steps forward, one step back. Trends Mol Med 2010, 16:203-209.

2. Friedenstein AJ, Gorskaja JF, Kulagina NN: Fibroblast precursors in normal and irradiated mouse hematopoietic organs. Exp Hematol 1976, 4:267-274.

3. Prockop DJ: Repair of tissues by adult stem/progenitor cells (MSC): controversies, myths, and changing paradigms. Mol Ther 2009, 17:939-946

4. Wu JC, Abraham MR, Kraitchman DL: Current perspectives on imaging cardiac stem cell therapy. J Nucl Med 2010, 51(Suppl 1):128S-136S.

5. Toma C, Wagner WR, Bowry S, Schwartz A, Villanueva F: Fate of culture-expanded mesenchymal stem cells in the microvasculature: in vivo observations of cell kinetics. Circ Res 2009, 104:398-402.

6. Barbash IM, Chouraqui P, Baron J, et al: Systemic delivery of bone marrow-derived mesenchymal stem cells to the infarcted myocardium: feasibility, cell migration, and body distribution. Circulation 2003, 108:863-868.

7. Merx MW, Zernecke A, Liehn EA, et al: Transplantation of human umbilical vein endothelial cells improves left ventricular function in a rat model of myocardial infarction. Basic Res Cardiol 2005, 100:208-216.

8. Bergwerf I, De Vocht N, Tambuyzer B, et al: Reporter gene-expressing bone marrow-derived stromal cells are immune-tolerated following implantation in the central nervous system of syngeneic immunocompetent mice. BMC Biotechnol 2009, 9:1.

9. Ronsyn MW, Daans J, Spaepen G, et al: Plasmid-based genetic modification of human bone marrow-derived stromal cells: analysis of cell survival and transgene expression after transplantation in rat spinal cord. BMC Biotechnol 2007, 7:90.

10. Swijnenburg RJ, Schrepfer S, Govaert JA, et al: Immunosuppressive therapy mitigates immunological rejection of human embryonic stem cell xenografts. Proc Natl Acad Sci U S A 2008, 105:12991-12996.

11. Swanger, Neuhuber B, Himes BT, Bakshi A, Fischer I: Analysis of allogeneic and syngeneic bone marrow stromal cell graft survival in the spinal cord. Cell Transplant 2005, 14:775-786.

12. Zangi $L$, Margalit $R$, Reich-Zeliger $S$, et al: Direct imaging of immune rejection and memory induction by allogeneic mesenchymal stromal cells. Stem Cells 2009, 27:2865-2874.

13. Liew A, O'Brien T: Therapeutic potential for mesenchymal stem cell transplantation in critical limb ischemia. Stem Cell Res Ther 2012, 3:28.

14. Gao J, Dennis JE, Muzic RF, Lundberg M, Caplan Al: The dynamic in vivo distribution of bone marrow-derived mesenchymal stem cells after infusion. Cells Tissues Organs 2001, 169:12-20.

15. Daldrup-Link H, E Rudelius $M$, et al: Cell tracking with gadophrin-2: a bifunctional contrast agent for MR imaging, optical imaging, and fluorescence microscopy. Eur J Nucl Med Mol Imaging 2004, 31:1312-1321.

16. Schrepfer S, Deuse T, Reichenspurner H, Fischbein MP, Robbins RC, Pelletier MP: Stem cell transplantation: the lung barrier. Transplant Proc 2007, 39:573-576.

17. Amsalem $Y$, Mardor $Y$, Feinberg MS, et al: Iron-oxide labeling and outcome of transplanted mesenchymal stem cells in the infarcted myocardium. Circulation 2007, 116:138-145.

18. Kang WJ, Kang HJ, Kim HS, Chung JK, Lee MC, Lee DS: Tissue distribution of 18F-FDG-labeled peripheral hematopoietic stem cells after intracoronary administration in patients with myocardial infarction. J Nucl Med 2006, 47:1295-1301.

19. Reekmans KP, Praet J, De Vocht N, et al: Clinical potential of intravenous neural stem cell delivery for treatment of neuro-inflammatory disease in mice? Cell Transplant 2010, 20:851-869.

20. Harting MT, Jimenez F, Xue H, Fischer UM, Baumgartner J, Dash PK, Cox CS: Intravenous mesenchymal stem cell therapy for traumatic brain injury. J Neurosurg 2009, 110:1189-1197.
21. Pittenger MF, Martin BJ: Mesenchymal stem cells and their potential as cardiac therapeutics. Circ Res 2004, 95:9-20.

22. Song H, Song BW, Cha MJ, Choi IG, Hwang KC: Modification of mesenchymal stem cells for cardiac regeneration. Expert Opin Biol Ther 2010, 10:309-319.

23. Liu H, Liu S, Li Y, et al: The role of SDF-1-CXCR4/CXCR7 axis in the therapeutic effects of hypoxia-preconditioned mesenchymal stem cells for renal ischemia/reperfusion injury. PLoS One 2012, 7:e34608.

doi:10.1186/1472-6750-12-93

Cite this article as: Everaert et al: Multimodal in vivo imaging reveals limited allograft survival, intrapulmonary cell trapping and minimal evidence for ischemia-directed BMSC homing. BMC Biotechnology 2012 12:93.

\section{Submit your next manuscript to BioMed Central and take full advantage of:}

- Convenient online submission

- Thorough peer review

- No space constraints or color figure charges

- Immediate publication on acceptance

- Inclusion in PubMed, CAS, Scopus and Google Scholar

- Research which is freely available for redistribution 